\title{
Developing a Discriminating Dissolution Procedure for a Dual Active Pharmaceutical Product with Unique Solubility Characteristics
}

\author{
Lagace, M. ${ }^{1,2}$, Gravelle, L. ${ }^{2}$, Di Maso, M. ${ }^{2}$ and McClintock, S. ${ }^{2}$
}

e-mail:marylene_lagace@merck.com

\begin{abstract}
Regulatory agencies often consider USP dissolution methods developed using the slowest paddle speed (50 rpm) to represent the most appropriate operating condition since they tend to produce the steepest drug release profiles. A steep drug release profile is frequently assumed to provide optimum discriminating power either to distinguish small variations in the tablet manufacturing process or to detect stability changes on storage. However, for certain tablet formulations it has been demonstrated that drug release profiles generated at $50 \mathrm{rpm}$ can in fact be a reflection of system artifacts rather than a discriminatory tool. Higher paddle speeds which result in flatter drug release profiles can, in some cases, more accurately reflect true formulation changes.

This point is highlighted in the description of the development of a dissolution method for a compressed tablet containing two active pharmaceutical ingredients (API). The selection of dissolution medium for a tablet with two APIs having very different solubility properties is detailed. The effects of paddle speed on system performance and method discriminating power are critically evaluated.
\end{abstract}

\section{Introduction}

D issolution testing is a required test currently used to demonstrate the performance of all solid oral dosage forms in which absorption of the drug is necessary for the product to exert a therapeutic effect (1). The challenge for scientists working in a research and development environment is to develop a procedure that can not only guide the formulation development process but can also be used as a regulatory test to detect manufacturing deviations and to ensure product consistency at release and over the product's shelf life. The test must be rugged and reproducible and highlight only significant changes in product performance. The robustness of the procedure is particularly important since calibrated dissolution baths are allowed a variation of $\pm 2 \mathrm{rpm}$ in the rotational speed of the apparatus. If a formulation is sensitive to small changes in rotational speed, then observed changes in the dissolution profile may simply reflect allowable instrument variation. The development of a dissolution procedure involves selecting the dissolution media, apparatus and agitation rate appropriate to the product.

The solubility of the active ingredient(s) is one of the key aspects in the screening of possible dissolution media. USP favors media related to physiological conditions, for example buffer solutions or diluted $\mathrm{HCl}(0.01 \mathrm{~N})$ (2). The dissolution characteristics of the formulation are to be evaluated over the physiologic $\mathrm{pH}$ range of 1.2 to 7.5 (1). For water-insoluble and sparingly water soluble drug products, use of a surfactant such as sodium lauryl sulfate is recommended (3).
To ensure good mixing of the drug and excipients in the dissolution vessel, a suitable apparatus and rotational speed should be selected. The basket method (Apparatus 1 ) is routinely used for capsule formulations at agitation speeds of 50 and $100 \mathrm{rpm}$, while the paddle method (Apparatus 2) is used mostly for tablets dosage forms at 50 and $75 \mathrm{rpm}$ (1). In general, mild agitation conditions should be maintained during dissolution testing to allow maximum discriminatory power (4). In most cases, the dissolution apparatus tends to become less discriminating when operated at faster speeds that result in a flatter drug release profile. However, for certain tablet formulations, the increased paddle speed results in a method with a higher discriminating power by reducing the variability of the data. Use of a low rotation speed could result in a lack of robustness in the data due to poor hydrodynamics in the dissolution vessel and can become more a reflection of system artifacts such as coning rather than true formulation changes. Visual observations are especially useful during method development to understand the behavior of the tablet in the dissolution vessel. The agitation speed providing optimum hydrodynamics in the vessel can be determined by comparison of the dissolution profiles obtained by making small variations in paddle speed (robustness experiments) as well as by challenging the testing procedure through the use of mismanufactured tablets (discriminatory power experiments). The final dissolution procedure should be robust and should be able to distinguish small but real changes in the product formulation.
${ }^{1}$ Corresponding author, Department of Pharmaceutical Research and Development, Merck Frosst Canada \& Co., 16711 Trans Canada Hwy., Kirkland, Quebec, $\mathrm{H} 9 \mathrm{H} 3 \mathrm{~L} 1 \mathrm{Canada}$

Telephone: 514-428-3783, Fax: 514-428-2677, e-mail address: marylene_lagace@merck.com

\author{
${ }^{2}$ Department of Pharmaceutical Research and Development, \\ Merck Frosst Canada \& Co., 16711 Trans Canada Hwy., Kirkland, Quebec, \\ H9H 3 L 1 Canada
}


A

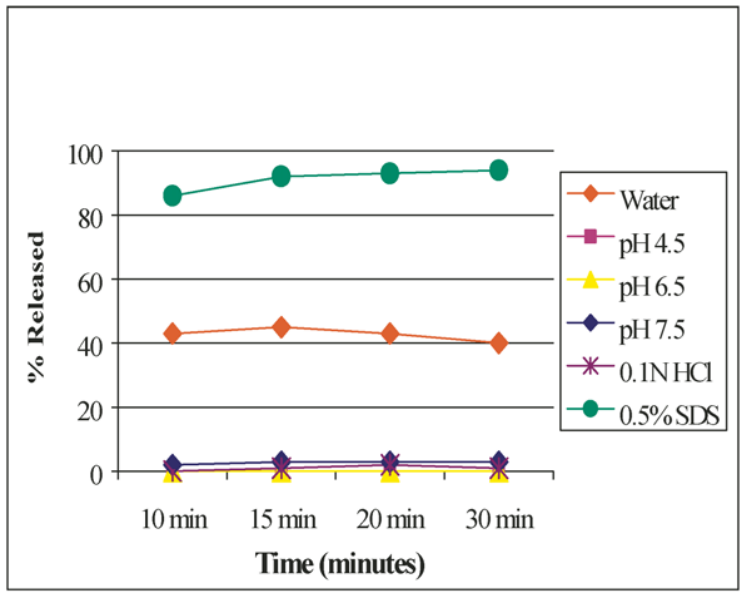

B

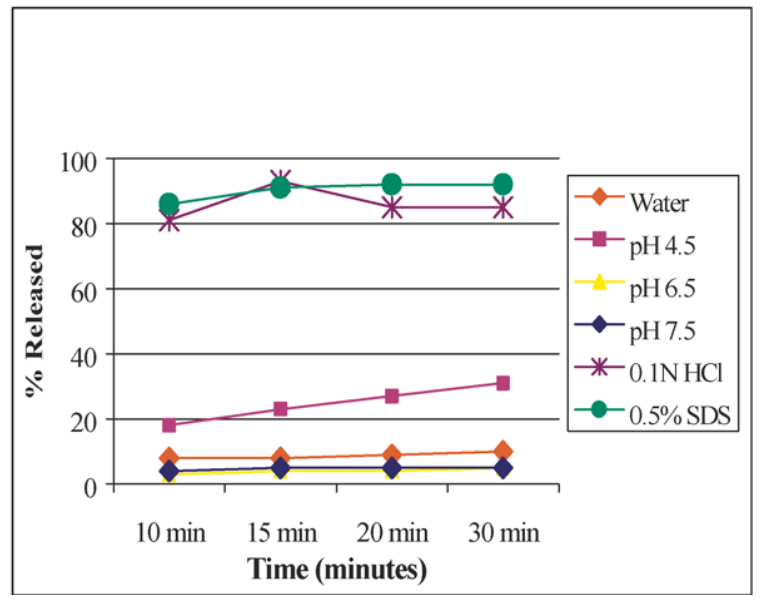

Figure 1. Dissolution rate of (A) Active $A$ and (B) Active B in various media using USP Apparatus II at 50 rpm and $37^{\circ} \mathrm{C}$

A

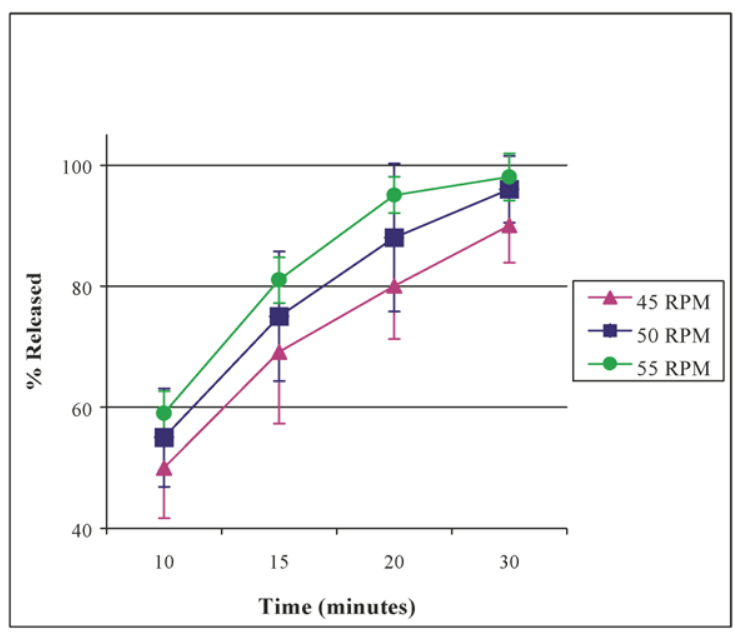

B

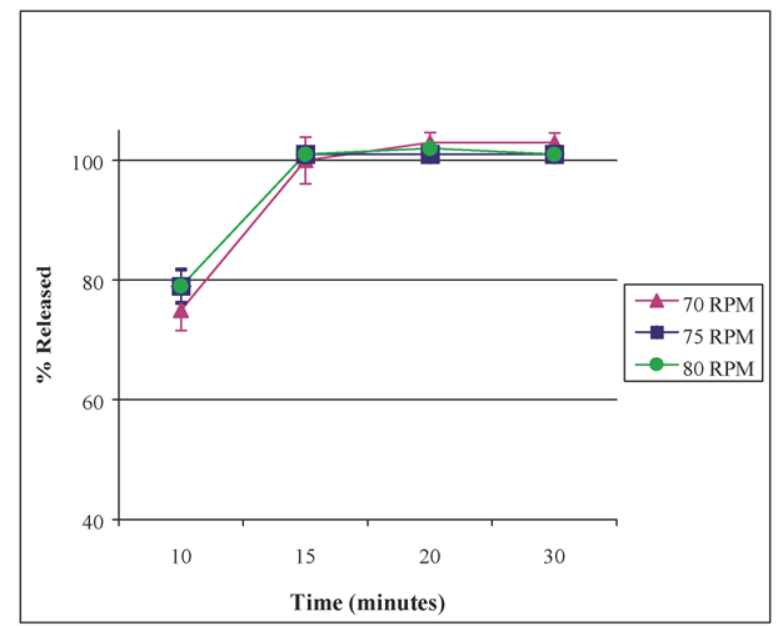

Figure 2. Dissolution of Active A at (A) $50 \pm 5 \mathrm{rpm}$ and (B) $75 \pm 5 \mathrm{rpm}$

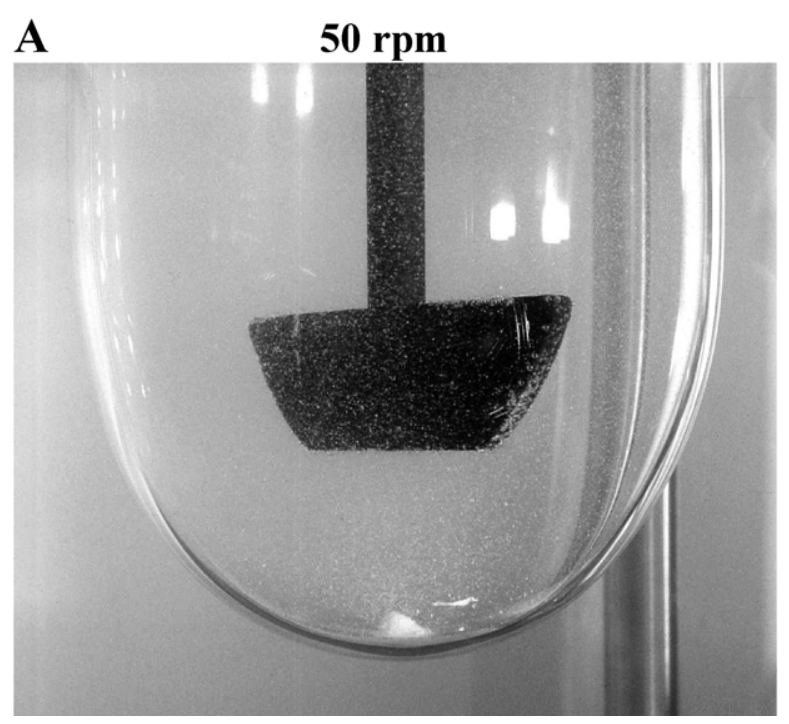

B

$75 \mathrm{rpm}$

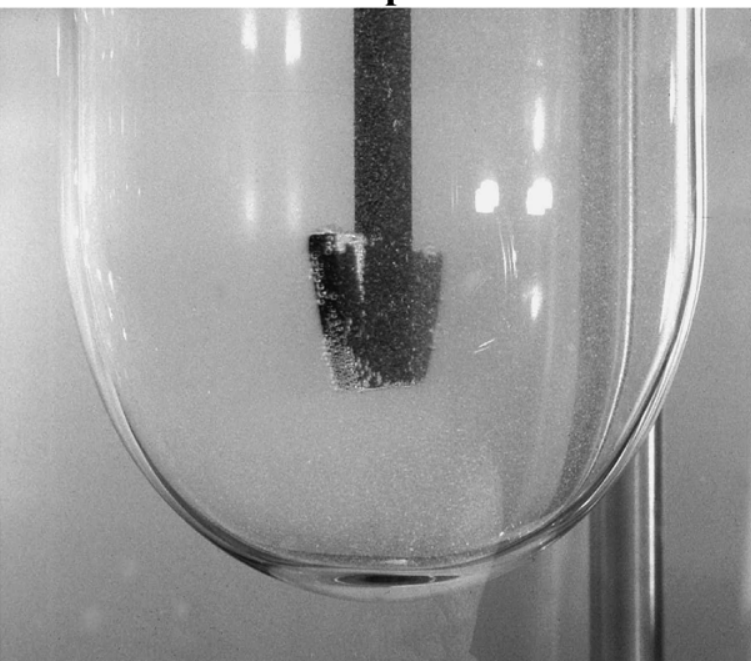

Figure 3. Visual observation of the dissolution of Compound $A / B$ at (A) $50 \mathrm{rpm}$ and (B) $75 \mathrm{rpm}$ after $15 \mathrm{~min}$ of paddle rotation 


\section{Experimental \\ Reagents}

All preparations (dissolution media and mobile phase) were carried out using the following reagents: Milli-Q grade water, hydrochloric acid $(\mathrm{HCl})$, potassium biphthalate $\left(\mathrm{KHC}_{6} \mathrm{H}_{4}(\mathrm{COO})_{2}\right.$, reagent grade), potassium phosphate $\left(\mathrm{KH}_{2} \mathrm{PO}_{4}\right.$, reagent grade), sodium lauryl sulfate (SDS; $\mathrm{CH}_{3}\left(\mathrm{CH}_{2}\right)_{10} \mathrm{CH}_{2} \mathrm{OSO}_{3} \mathrm{Na}-99 \%$ pure; $\mathrm{ACS}$ grade), sodium phosphate monobasic monohydrate $\left(\mathrm{NaH}_{2} \mathrm{PO}_{4} \bullet \mathrm{H}_{2} \mathrm{O}\right.$, reagent grade).

Buffer solutions were prepared as described in the United States Pharmacopeia (USP, Edition 23), Reagents, Indications, and Solutions section.

\section{Dissolution Methodology}

Experiments were carried out using a manual Distek Dissolution System equipped with paddles (USP Apparatus II) and amber Distek or Vankel dissolution vessels. Clear Vankel PEAK ${ }^{\mathrm{TM}}$ vessels were also used to investigate coning. A dissolution volume of $900 \mathrm{~mL}$ was used at a temperature of $37 \pm 0.5^{\circ} \mathrm{C}$. The procedure used paddles at $50 \pm 5 \mathrm{rpm}$ and $75 \pm 5 \mathrm{rpm}$. Samples were taken at 10, 15, 20 and 30 minutes. A minimum of 6 vessels were sampled for each analysis.

\section{HPLC Methodology}

Quantitation was performed with a Hewlett Packard/Agilent 1100 series High Performance Liquid Chromatograph (HPLC). The method utilizes a Zorbax XDB C18, $5 \mathrm{~cm} \times$ 4.6-mm internal diameter, 3.5- $\mu \mathrm{m}$ particle size HPLC column with a mobile phase composed of $38 \% 25 \mathrm{mM}$ sodium phosphate buffer $\mathrm{pH} 7.0$ and $62 \%$ acetonitrile, a flow rate of $2.0 \mathrm{~mL} / \mathrm{min}$ (run time of 2 minutes), a column temperature of $35^{\circ} \mathrm{C}$ and an injection volume of $10 \mu \mathrm{L}$. Detection of both actives was by UV at a wavelength of $250 \mathrm{~nm}$.

\section{Results and Discussion \\ Medium Selection}

In selecting a medium for dissolution testing of Compound $A / B$, the solubility characteristics of the individual active ingredients must be considered. Active $A$ is freely soluble in water (>140 mg/ml solubility). However, equilibrium solubility measurements are problematic. With increasing concentration in solution, Active A initially forms micelles, then larger organized structures and finally a gelatin-like mass that exhibits some of the properties of liquid crystals. Active A's solubility is dependent on its surface-active properties and its ability to self-associate in concentrated solutions. However, in dilute aqueous solutions, typical of concentrations found in dissolution procedures, self-association is less likely and the active precipitates from solution as the free acid. Both $\mathrm{pH}$ and ionic strength affect its solubility. The native $\mathrm{pH}$ of a $1 \%$ solution of Active $A$ is approximately 9.5. The free acid precipitates when $\mathrm{pH}$ is less than 8.

Active $B$ is a crystalline hydrophobic compound with practically no aqueous solubility $(0.0021 \mathrm{mg} / \mathrm{mL})$. Its solu- bility increases at low $\mathrm{pH}$ to $26.8 \mathrm{mg} / \mathrm{ml}$ in $0.1 \mathrm{~N} \mathrm{HCl}$, due to the formation of the protonated base. It has a solubility of $0.0017 \mathrm{mg} / \mathrm{ml}$ in $0.1 \mathrm{~N} \mathrm{NaOH}$. The addition of surfactants increases the aqueous solubility to $1.2 \mathrm{mg} / \mathrm{ml}$ in $0.5 \%$ SDS, a 600 -fold increase relative to that in water. In $1 \%$ SDS, the equilibrium solubility of Active $B$ is $2.8 \mathrm{mg} / \mathrm{ml}$.

The choice of dissolution medium to accommodate both active compounds is limited. According to the USP, dissolution medium may be water, a buffered aqueous solution (typically $\mathrm{pH} 4$ to 8 ) or a dilute acid $(0.001$ to $0.1 \mathrm{~N}$ $\mathrm{HCl}$ ). Surfactants and electrolytes may also be added to aid in the solubilization of the active ingredient(s). Screening of the following media was carried out using USP Apparatus II at $50 \mathrm{rpm}$ and $37^{\circ} \mathrm{C}$ : (a) water; (b) USP buffer $\mathrm{pH} 4.5$; (c) USP buffer $\mathrm{pH} 6.5$; (d) USP buffer $\mathrm{pH} 7.5$; (e) $0.1 \mathrm{~N} \mathrm{HCl}$ and (f) $0.5 \%$ SDS in water. The results of this screening are shown in Figure 1.

Active $A$ is almost insoluble in the 3 USP buffers, $0.1 \mathrm{~N}$ $\mathrm{HCl}$ and water. Active $B$ has very low solubility in water and the 3 USP buffers. In $0.1 \mathrm{~N} \mathrm{HCl}$, Active B is more soluble, but only $0.5 \%$ SDS provides adequate solubility for both active ingredients.

\section{Apparatus and Paddle Speed Selection}

The apparatus and rotational speed selected must provide adequate mixing to disperse the drug product in the media and to provide a homogeneous mixture for sampling, while maintaining the discriminatory power of the dissolution procedure.

USP Apparatus II was chosen due to its acceptance as a standard procedure for tablet formulations. Paddle speeds of 50 and $75 \mathrm{rpm}$ were evaluated with samples taken after 10,15, 20 and 30 minutes of paddle rotation. In order to demonstrate method robustness, dissolutions were performed using paddle speeds of $50 \pm 5 \mathrm{rpm}$ and $75 \pm 5 \mathrm{rpm}$. The graphs in Figure 2 show the dissolution profiles of Active A at both 50 and 75 rpm. Active B produced similar profiles.

The dissolution at $50 \mathrm{rpm}$ provides a steeper profile than at $75 \mathrm{rpm}$. However, the high variability observed by varying the paddle speeds $\pm 5 \mathrm{rpm}$ at $50 \mathrm{rpm}$ demonstrates a lack of method robustness. At $75 \pm 5 \mathrm{rpm}$, the profile is flatter, but more robust as shown by the lower variability in the data.

In an attempt to understand the origin of the method variability at 50 rpm, a visual observation of the tablet behavior in the dissolution vessel was performed at both 50 and 75 rpm. The following photographs (Figure 3) were taken of the dissolution procedure after 15 minutes of paddle rotation.

At 50 rpm, the tablet collapses into a "cone" after disintegration, which traps the drug in a pile of insoluble excipients in the bottom of the dissolution vessel. This results in incomplete dissolution. "Cone" formation is a system artifact observed when poor hydrodynamics, and thus poor solution mixing, exist in the dissolution vessel. This common phenomenon is associated with certain types of tablet formulations and with poor hydrodynamics associated with USP Apparatus II at a paddle speed of $50 \mathrm{rpm}$ 


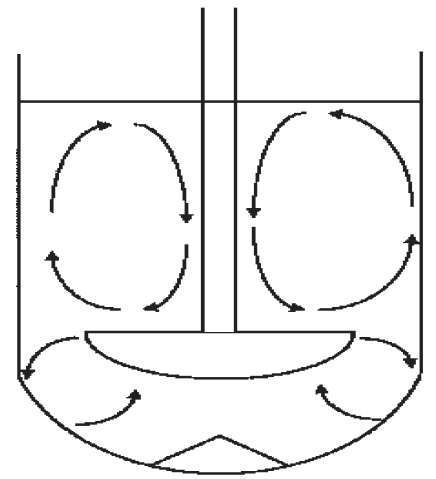

Figure 4. Schematic diagram of flow patterns leading to coning in a dissolution vessel

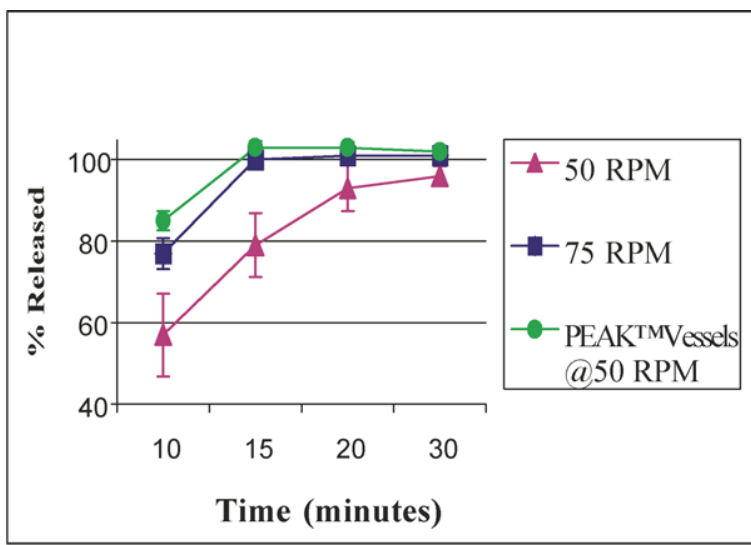

Figure 5. Dissolution of Active A in PEAK ${ }^{\mathrm{TM}}$ vessels at $50 \mathrm{rpm}$

(refer to Figure 4). This "cone" formation explains the high variability in the dissolution data observed at $50 \pm 5 \mathrm{rpm}$.

Although dissolution methods exhibiting coning behaviour may produce a more "classic" profile, the inherent risks outweigh potential benefits from such a system. For example, there is an implicit assumption that the formation of the cone will be consistent over time and that it will not be affected by minor variations in equipment.

When the paddle speed is increased to $75 \mathrm{rpm}$, the increased turbulence in the vessel disrupts the "cone" for- mation thus providing sufficient hydrodynamics to expose all tablet granules to the dissolution medium. The dissolution profile subsequently obtained more accurately reflects the dissolution of the tablet, not system hydrodynamics, and demonstrates a more rugged test procedure. To confirm these observations, dissolution testing was performed at 50 rpm using PEAK ${ }^{\mathrm{TM}}$ vessels, which, due to the presence of a protrusion at the bottom of the vessel, inhibit "cone" formation. Figure 5 shows that the use of PEAK ${ }^{\mathrm{TM}}$ vessels at $50 \mathrm{rpm}$ gives low variability and a similar profile to that obtained at $75 \mathrm{rpm}$ in regular vessels.

To challenge the ability of the dissolution procedure to demonstrate sufficient discriminating power, tablets were purposely mis-manufactured with modified excipient composition and tablet hardness and evaluated using both 50 and 75-rpm paddle speeds. In Figure 6, the resulting profiles were plotted along with the robustness profiles obtained by varying the rotational speed by $\pm 5 \mathrm{rpm}$. At $50 \mathrm{rpm}$, the dissolution profile of the mis-manufactured tablets cannot be distinguished from that of properly manufactured tablets. The change in profile attributable to mis-manufacture of the tablets is overwhelmed by the method variability. At 75 rpm, the mis-manufactured tablets give a depressed dissolution profile that is readily distinguished by the method. The reduced power to detect manufacturing defects at 50 rpm is due to the compromised method robustness inherent in dissolution methods displaying significant "coning".

The ability of the dissolution procedure to demonstrate sufficient discriminating power and sensitivity at $75 \mathrm{rpm}$ was also tested on a highly stressed sample. Tablets stored in open containers for 4 weeks at $40^{\circ} \mathrm{C} / 75 \%$ relative humidity were tested and compared to tablets stored in a market package at ambient conditions. As shown in Figure 7, the dissolution procedure at $75 \mathrm{rpm}$ is able to discriminate between the profiles that are produced by stressed and unstressed tablets.

Based on the visual observations, robustness evaluation and evaluation of sensitivity to manufacturing and storage changes, the dissolution procedure at $75 \mathrm{rpm}$ more accurately reflects the dissolution profile of the product. The procedure is more rugged and reliable than testing
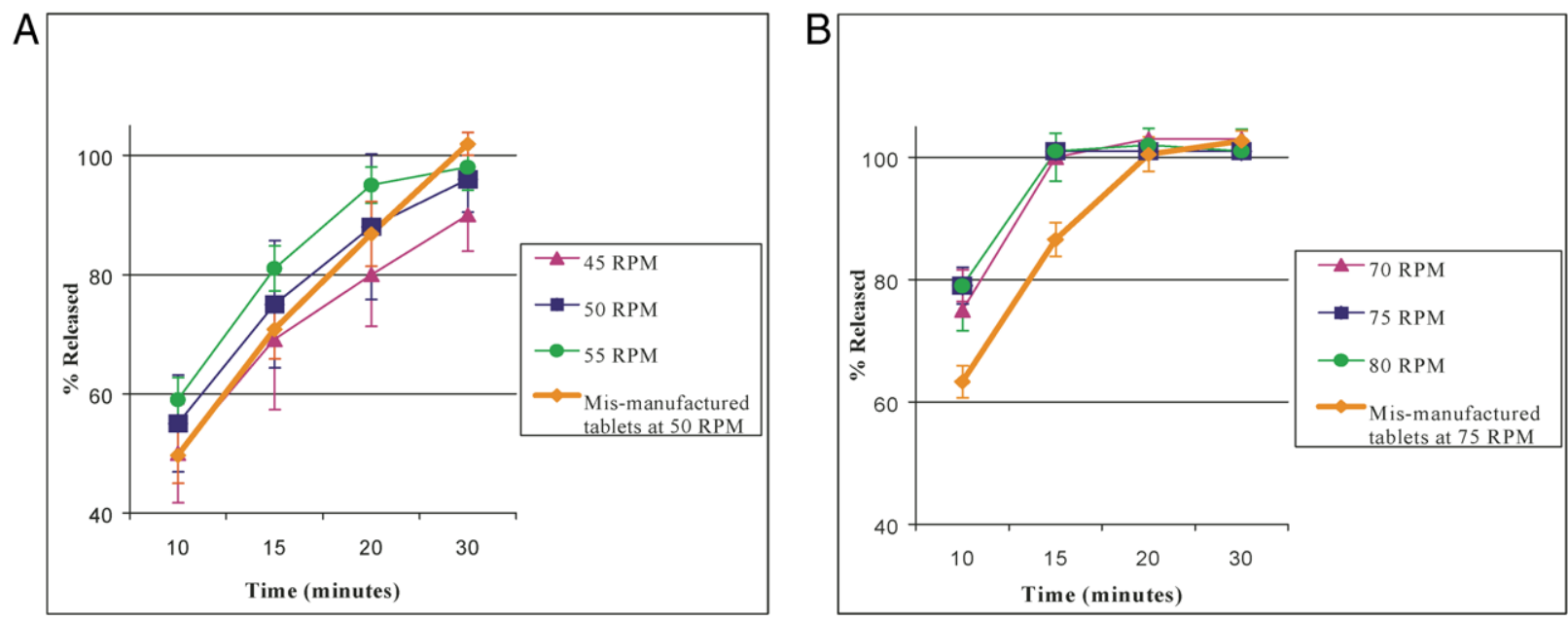

Figure 6. Dissolution of mis-manufactured tablets at (A) $50 \mathrm{rpm}$ and (B) $75 \mathrm{rpm}$ 


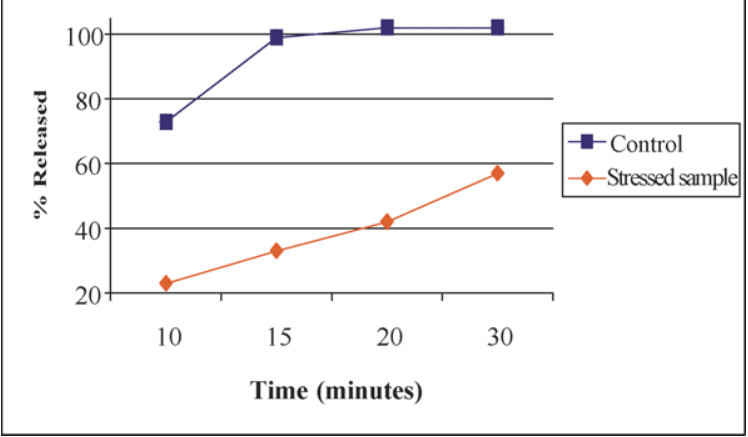

Figure 7: Dissolution of stressed vs. unstressed tablets at $75 \mathrm{rpm}$.

performed at $50 \mathrm{rpm}$. Therefore, the rotational speed of $75 \mathrm{rpm}$ was chosen for the dissolution procedure.

\section{Conclusions}

In general, use of the slowest calibrated paddle speed $(50 \mathrm{rpm})$ results in a method with a steeper drug release profile, typically leading to a higher discriminating power. However, for this formulation the use of a slower rotation speed resulted in a lack of robustness and the dissolution became more a reflection of system artifacts, such as coning, than true formulation changes. Visual observations were especially useful during method development, when understanding the physical behavior of the tablets in the dissolution vessel was necessary.
The agitation speed providing optimum hydrodynamics in the vessel was determined through comparison of the dissolution profiles obtained from small variations in paddle speed as well as by challenging the testing procedure with the use of mis-manufactured tablets. Although the method using a paddle speed of $50 \mathrm{rpm}$ produced a more "classic" dissolution profile, its ability to discriminate between manufacturing changes was overwhelmed by lack of method robustness. A paddle speed of $75 \mathrm{rpm}$ not only produced an expected increase in robustness but also provided a procedure with superior discriminatory power. The final dissolution procedure selected is robust and able to distinguish small changes in the product formulation.

\section{Bibliography}

1. Gray, V.A., Brown, C.K., Dressman, J.B. and Leeson, J. 2001. A New General Information Chapter on Dissolution. Pharmacopeial Forum 27(6):3432-3439

2. FDA. August 1997. Guidance for Industry, Dissolution Testing of Immediate Release Solid Oral Dosage Forms.

3. Shah, V.P., Noory, A., Noory, C., McCullough, B., Clarke, S., Everett, R., Naviasky, H., Srinivasan, B.N., Fortman, D. and Skelly, J.P. 1995. In Vitro Dissolution of Sparingly Water-Soluble Drug Dosage Forms. International Journal of Pharmaceutics 125:99-106.

4. NF XX/USP XXV. 2002. The United States Pharmacopeia Convention, Inc., Rockville, MD, p. 2160-2165. 